\title{
RINGS IN WHICH CERTAIN RIGHT IDEALS ARE DIRECT SUMMANDS OF ANNIHILATORS
}

\author{
YIQIANG ZHOU
}

(Received 15 May 2000; revised 8 October 2001)

Communicated by Jie Du

\begin{abstract}
This paper is a continuation of the study of the rings for which every principal right ideal (respectively, every right ideal) is a direct summand of a right annihilator initiated by Stanley $\mathbf{S}$. Page and the author in $[20,21]$.
\end{abstract}

2000 Mathematics subject classification: primary 16D50, $16 \mathrm{P} 60$.

\section{Introduction}

In this paper, we continue the study of left AP-injective and left AGP-injective rings which were introduced and discussed in [20]. Following [20], a ring $R$ is called left $A P$-injective if every principal right ideal is a direct summand of a right annihilator, and the ring $R$ is called left AGP-injective if, for any $0 \neq a \in R$, there exists $n>0$ such that $a^{n} \neq 0$ and $a^{n} R$ is a direct summand of $\mathbf{r l}\left(a^{n}\right)$. Recall that a ring $R$ is left principally injective ( $\boldsymbol{P}$-injective) if every principal right ideal is a right annihilator, and the ring $R$ is left generalized principally injective (GP-injective) if, for any $0 \neq a \in R$, there exists $n>0$ such that $a^{n} \neq 0$ and $a^{n} R$ is a right annihilator. The detailed discussion of left P-injective and left GP-injective rings can be found in $[3,7,12,15,16,17,22,23,24,26]$. Clearly, every left AP-injective ring is left P-injective and every left AGP-injective ring is left GP-injective. But there exist left AP-injective rings which are not left GP-injective [20]. In fact, a left AP-injective ring is not necessarily a left mininjective ring. (The ring $R$ is left mininjective if,

The author was supported in part by the NSERC grant OGP0194196.

(C) 2002 Australian Mathematical Society 1446-7887/2000\$A2.00+0.00 
for any minimal left ideal $R a, a R$ is a right annihilator [18], and every left GPinjective ring is left mininjective.) In [20], several results which are known for left P-injective (respectively, left GP-injective) rings were shown to hold for left APinjective (respectively, left AGP-injective) rings. It has been noted that it is unknown whether there exists a left GP-injective ring that is not left P-injective (see [6, 24]). This may put a bit more weight on our excuse for carrying on the study of the left AGP-injective rings. In this paper, we discuss left AGP-injective rings with various chain conditions.

It is well known that a ring $R$ is quasi-Frobenius (QF) if and only if $R$ is left selfinjective and left (or right) noetherian. In [9], Faith proved that any left self-injective ring satisfying the ACC on left annihilators is QF. Bjồrk [2] extended this result from a left self-injective ring to a left $\mathrm{f}$-injective ring, and then Rutter [23] further proved that, if $R$ satisfies the ACC on left annihilators, then $R$ is QF if and only if $R$ is left 2-injective, where the ring $R$ is called left f-injective (respectively, left 2-injective) if, for any finitely generated (respectively, 2-generated) left ideal $I$ of $R$, every $R$ homomorphism from $I$ to $R$ extends to an $R$-homomorphism from $R$ to $R$. Note that a left $f$-injective rings need not be left self-injective, and a left $P$-injective ring need not be left 2-injective. It was also proved in [23] that any left $P$-injective ring satisfying the ACC on left annihilators is right artinian. The latter result was extended from a left P-injective ring to a left GP-injective ring in Chen and Ding [7]. It is clear, by Rutter's example in [23], that a left P-injective ring satisfying the ACC on left annihilators need not be left artinian, and hence not be QF. The main result in Section 2 states that a left AGP-injective ring with the ACC on left annihilators is always semiprimary, but is not necessarily right artinian.

A ring is called a right dual ring if every right ideal is a right annihilator. The study of right noetherian, right dual rings was initiated by Johns [14], and continued by Faith and Menal in $[10,11]$ where they gave a counterexample to Johns' result that every right noetherian, right dual ring is right artinian. Recently, Gómez Pardo and Guil Asensio [12] proved that if $R$ is right noetherian and left P-injective, then $J(R)$ is nilpotent and $\mathrm{I}(J(R))$ is essential both as a left and a right ideal of $R$, and this result allows them to show that every left Kasch, right noetherian and left P-injective ring is right artinian. In Section 2, among other things, we prove that, for a right noetherian and left AGP-injective ring $R, J(R)$ is nilpotent and $\mathrm{I}(J(R))$ is essential both as a left and a right ideal of $R$. As a corollary of this, we show that every right noetherian, left AGP-injective ring with right (GC2) is right artinian.

In Section 3, we consider right quasi-dual rings. A ring $R$ is called right quasi-dual if every right ideal of $R$ is a direct summand of a right annihilator [21]. The right quasi-dual rings form an interesting class of left AP-injective rings. In Section 3, it is proved that, for a right quasi-dual ring, $J(R)=\mathbf{r}\left(S_{r}\right), S_{r}=\mathbf{r}\left(Z_{r}\right)$ and $\mathbf{I}(J(R))$ is essential in ${ }_{R} R$. Consequently, for a two-sided quasi-dual ring $R$, the left socle 
coincides with the right socle and is essential both as a left and a right ideal of $R$. We also improve a result of [21] by showing that a ring $R$ is a two-sided PF-ring if and only if every right Goldie torsion $R$-module is cogenerated by $R_{R}$ and every left Goldie torsion $R$-module is cogenerated by ${ }_{R} R$.

Throughout, $R$ is an associative ring with identity and modules are unitary. We use $M_{R}$ (respectively, ${ }_{R} M$ ) to indicate that $M$ is a right (respectively, left) module over $R$. For a subset $X$ of $R, \mathbf{l}(X)$ (respectively, $\mathbf{r}(X)$ ) is the left (respectively, right) annihilator of $X$ in $R$, and we write $\mathrm{l}(x)$ (respectively, $\mathbf{r}(x))$ for $\mathrm{I}(\{x\})$ (respectively, $\mathbf{r}(\{x\}))$ when $x \in R$. The left socle, right socle, left singular ideal, right singular ideal and Jacobson radical of $R$ are denoted by $S_{l}, S_{r}, Z_{l}, Z_{r}$ and $J(R)$, respectively. For a submodule $N$ of $M$, we use $N \leq_{e} M$ to mean that $N$ is essential in $M$.

\section{Left AGP-injective rings with left chain conditions}

Following [20], the ring $R$ is left AP-injective if, for any $a \in R, a R$ is a direct summand of $\operatorname{rl}(a)$, and $R$ is left AGP-injective if, for any $0 \neq a \in R$, there exists $n>0$ such that $a^{n} \neq 0$ and $a^{n} R$ is a direct summand of $\mathbf{r l}\left(a^{n}\right)$. Every left P-injective ring is left AP-injective and every left GP-injective ring is left AGP-injective. The rings $R$ in [21, Examples 2.3,2.4] are commutative AP-injective rings, but not mininjective and hence not GP-injective.

In this section, we prove several results of left AGP-injective rings with some chain conditions on left ideals.

A module $M$ is said to satisfy the generalized C2-condition (or (GC2)) if, for any $N \subseteq M$ and $N \cong M, N$ is a summand of $M$. Note that the GC2-condition is the same as the $(*)$-condition in [20, page 713 ].

LEMMA 1.1. Let ${ }_{R} M$ satisfy (GC2). If $M$ is finitely dimensional, then $\operatorname{End}(M)$ is semilocal.

Proof. Let $\sigma: M \rightarrow M$ be a monomorphism. Then $M=\sigma(M) \oplus N$ for some $N \subseteq M$. It must be that $N=0$ since $M$ is finitely dimensional. So, $\sigma$ is an isomorphism. Therefore, $M$ satisfies the assumptions in Camps-Dicks [5, Theorem 5], and so $\operatorname{End}(M)$ is semilocal.

The next corollary extends [21, Proposition 2.12].

COROLLARY 1.2. Let $R$ be a left AGP-injective ring.

(1) If ${ }_{R} R$ is of finite Goldie dimension, then $R$ is semilocal.

(2) $R$ is left noetherian if and only if $R$ is left artinian. 
ProOf. (1). By [20, Proposition 2.13], ${ }_{R} R$ satisfies (GC2). Since ${ }_{R} R$ has finite Goldie dimension, $R$ is semilocal by Lemma 1.1.

(2). If $R$ is left noetherian, then $R$ is semilocal by (1). By [20, Corollary 2.11], $J(R)$ is nilpotent. So, $R$ is left artinian.

\section{LEMMA 1.3 ([20]). If $R$ is a left AGP-injective ring, then $J(R)=Z_{l}$.}

LEMMA 1.4. Let $R$ be a left $A G P$-injective ring and $a \in R$. If $a \notin J(R)$ then there exists $r \in R$ such that the inclusion $\mathbf{I}(a) \subset \mathbf{l}(a-a r a)$ is proper.

ProOF. Let $a \in R$ but $a \notin J(R)$. By Lemma $1.3, a \notin Z_{l}$ and hence $\mathrm{I}(a)$ is not essential in ${ }_{R} R$. So, we have $\mathrm{l}(a) \cap I=0$ for some $0 \neq I \subseteq{ }_{R} R$. Take $0 \neq b \in I$. Then $b a \neq 0$. By the hypothesis, there exists $n>0$ such that $(b a)^{n} \neq 0$ and $\operatorname{rl}\left((b a)^{n}\right)=$ $(a b)^{n} R \oplus X$ where $X$ is a right ideal of $R$. Since $\mathbf{I}(a) \cap I=0, \mathbf{I}\left((b a)^{n}\right)=\mathbf{I}\left((b a)^{n-1} b\right)$. It follows that $(b a)^{n-1} b \in \mathbf{r l}\left((b a)^{n-1} b\right)=\operatorname{rl}\left((b a)^{n}\right)=(b a)^{n} R \oplus X$. Thus, there exists $r \in R$ such that $(b a)^{n-1} b=(b a)^{n} r+x$ where $r \in R$ and $x \in X$. This gives that $(b a)^{n-1} b(1-a r)=x$ and hence $(b a)^{n-1} b(a-a r a)=x a \in(b a)^{n} R \cap X$. It follows that $(b a)^{n-1} b(a-a r a)=0$. Let $c=a-a r a$. Then $\mathbf{l}(a) \subseteq \mathbf{l}(c)$. Since $(b a)^{n-1} b$ is in $\mathbf{I}(c)$ but not in $\mathbf{I}(a)$, the inclusion $\mathbf{I}(a) \subset \mathbf{I}(c)$ is proper.

The next result extends [7, Theorem 3.4, Corollary 3.6]. Following [1], a module $M$ is called finitely projective (respectively, singly projective) if, for each epimorphism $f: N \rightarrow M$ and each finitely generated (respectively, cyclic) submodule $M_{0}$ of $M$, there exists $g \in \operatorname{Hom}_{R}\left(M_{0}, N\right)$ such that the restriction of $g \circ f$ to $M_{0}$ is the identity on $M_{0}$.

THEOREM 1.5. The following are equivalent for a left AGP-injective ring $R$ :

(1) $R$ is a left Perfect ring.

(2) Every flat left $R$-module is finitely projective.

(3) Every flat left $R$-module is singly projective.

(4) For any infinite sequence $x_{1}, x_{2}, x_{3}, \ldots$ of elements in $R$, the chain $\mathrm{I}\left(x_{1}\right) \subseteq$ $\mathbf{l}\left(x_{1} x_{2}\right) \subseteq \mathbf{l}\left(x_{1} x_{2} x_{3}\right) \subseteq \cdots$ terminates.

Proof. (1) implies (2) and (2) implies (3) are obvious. (3) implies (4) is by [1, Corollary 25].

(4) implies (1). Firstly, we prove $R / J(R)$ is a von Neumann regular ring. For any $x \in R$, let $\bar{x}=x+J(R)$. Let $a_{1} \in R$ but $a_{1} \notin J(R)$. We want to show that $\bar{a}_{1}=\bar{a}_{1} \bar{x} \bar{a}_{1}$ for some $x \in R$. By Lemma 1.4, there exists $r_{1} \in R$ such that $\mathrm{I}\left(a_{1}\right) \subset \mathrm{I}\left(a_{2}\right)$ where $a_{2}=a_{1}-a_{1} r_{1} a_{1}$. If $a_{2} \in J(R)$, then $\bar{a}_{1}=\bar{a}_{1} \bar{r}_{1} \bar{a}_{1}$ and we are done. If $a_{2} \notin J(R)$, then, by Lemma 1.4, there exists $r_{2} \in R$ such that $\mathbf{I}\left(a_{2}\right) \subset \mathbf{I}\left(a_{3}\right)$ where $a_{3}=a_{2}-a_{2} r_{2} a_{2}$. The induction principle and the hypothesis ensure the existence of a positive integer 
$n$ and two sequences $\left\{a_{i}: i=1, \ldots, n+1\right\}$ and $\left\{r_{i}: i=1, \ldots, n\right\}$ of elements in $R$ such that $a_{n+1} \in J(R)$ and $a_{i+1}=a_{i}-a_{i} r_{i} a_{i}$ for $i=1, \ldots, n$. Thus, $\bar{a}_{n}=\bar{a}_{n} \bar{r}_{n} \bar{a}_{n}$. It follows that

$$
\begin{aligned}
\bar{a}_{n-1} & =\bar{a}_{n}+\bar{a}_{n-1} \bar{r}_{n-1} \bar{a}_{n-1} \\
& =\left(\bar{a}_{n-1}-\bar{a}_{n-1} \bar{r}_{n-1} \bar{a}_{n-1}\right) \bar{r}_{n}\left(\bar{a}_{n-1}-\bar{a}_{n-1} \bar{r}_{n-1} \bar{a}_{n-1}\right)+\bar{a}_{n-1} \bar{r}_{n-1} \bar{a}_{n-1} \\
& =\bar{a}_{n-1}\left[\left(\overline{1}-\bar{r}_{n-1} \bar{a}_{n-1}\right) \bar{r}_{n}\left(\overline{1}-\bar{a}_{n-1} \bar{r}_{n-1}\right)+\bar{r}_{n-1}\right] \bar{a}_{n-1},
\end{aligned}
$$

so $\bar{a}_{n-1}$ is also a regular element. Continuing this process, we see that $\bar{a}_{1}$ is a regular element.

Secondly, we prove that $Z_{l}$ is left T-nilpotent. Let $a_{i} \in Z_{l}$ for $i=1,2, \ldots$ We have a chain $\mathbf{I}\left(a_{1}\right) \subseteq \mathbf{I}\left(a_{1} a_{2}\right) \subseteq \cdots$. By our assumption, there exists $n>0$ such that $\mathbf{I}\left(a_{1} \cdots a_{n}\right)=\mathbf{I}\left(a_{1} \cdots a_{n} a_{n+1}\right)$. Thus, $\mathbf{I}\left(a_{n+1}\right) \cap R a_{1} \cdots a_{n}=0$. Since $\mathbf{I}\left(a_{n+1}\right)$ is essential in ${ }_{R} R$, we have $a_{1} \cdots a_{n}=0$, so $Z_{l}$ is left T-nilpotent. Therefore, by Lemma 1.3, we have proved that $R / J(R)$ is a von Neumann regular ring and $J(R)$ is left T-nilpotent. So, it suffices to show that $R / J(R)$ is an artinian semisimple ring. By [13, Corollary 2.16], we only need to show that $R / J(R)$ contains no infinite sets of nonzero orthogonal idempotents. This can be proved by arguing as in $[7$, page 2107].

COROLLARY 1.6. If $R$ is a left AGP-injective ring with ACC on left annihilators, then $R$ is semiprimary.

PROOF. It is well known that $Z_{l}$ is nilpotent for any ring $R$ with ACC on left annihilators. By Lemma 1.3 and Theorem 1.5, $R$ is semiprimary.

COROLLARY 1.7. Let $R$ be a left AGP-injective ring with ACC on left annihilators and $S_{r} \subseteq S_{l}$. Then $R$ is right artinian if and only if $S_{r}$ is a finitely generated right ideal of $R$.

Proof. By Corollary 1.6, $R$ is semiprimary. By [20, Corollary 2.7], $S_{l} \subseteq S_{r}$, and so $S=S_{l}=S_{r}$ by the hypothesis. Now the result follows from [4, Lemma 6].

A left GP-injective ring with the ACC on left annihilators is always right artinian [7, Theorem 3.7]. The ring $R$ [21, Example 2.4] is a commutative AP-injective ring with the ACC on annihilators, but $R$ is not artinian.

Recall that a ring $R$ is called left $\operatorname{Kasch}$ if $\mathbf{r}(K) \neq 0$ for every maximal left ideal $K$ of $R$.

COROLLARY 1.8. Let $R$ be a left AGP-injective ring with ACC on left annihilators. If every minimal right ideal is a right annihilator, then $R$ is right artinian. Moreover, $R$ is left artinian if and only if $S_{l}$ is finitely generated as a left ideal of $R$. 
PRoof. By Corollary 1.6, $R$ is semiprimary. By [18, Corollary 3.15], $R$ is right finite dimensional with $S_{r}=S_{l}$. Now, by [4, Lemma 6], $R$ is right artinian. The last assertion follows from [4, Lemma 6] again.

Now the following result, [7, Theorem 3.7], is an immediate corollary of the above:

COROLLARY 1.9 ([7]). Every left GP-injective ring with ACC on left annihilators is right artinian.

ProOF. If $R$ is a left GP-injective ring, then every minimal right ideal is a right annihilator. For, if $I$ is a minimal right ideal of $R$, then $I=e R$ where $e^{2}=e \in R$ or $I^{2}=0$. If $I=e R$, clearly $I$ is an annihilator. 'On the other hand, if $I=x R$ for some $x \in R$ with $I^{2}=0$, it follows from the definition of left GP-injectivity that $I=x R=\mathbf{r l}(I)$. Now the result follows from Corollary 1.8 .

\section{Left AGP-injective rings with right chain conditions}

In this section, we first consider right noetherian, left AGP-injective rings. We prove that, for a right noetherian, left AGP-injective ring $R, J(R)$ is nilpotent and $\mathbf{I}(J(R))$ is essential as a left and as a right ideal of $R$. As a corollary of this, we prove that every right noetherian, left AGP-injective ring $R$ such that $R_{R}$ satisfies (GC2) is right artinian. We next prove that every maximal left (respectively, right) annihilator of a semiprime left AGP-injective ring is a maximal left (respectively, right) ideal generated by an idempotent.

The next result extends [12, Theorem 2.7] from a left P-injective ring to a left AGP-injective ring.

THEOREM 2.1. Let $R$ be a right noetherian, and left AGP-injective ring. Then $J(R)$ is nilpotent and $\mathrm{I}(J(R))$ is essential both as a left and as a right ideal of $R$.

PROOF. Let $J=J(R)$. First we prove that $\mathrm{I}(J) \leq_{e} R$. Let $0 \neq x \in R$. Since $R$ is right noetherian, the non-empty set $\mathscr{F}=\left\{\mathbf{r}\left((a x)^{k}\right): a \in R, k>0\right.$ such that $\left.(a x)^{k} \neq 0\right\}$ has a maximal element, say $\mathbf{r}\left((y x)^{n}\right)$.

We claim that $(y x)^{n} J=0$. If not, then there exists $t \in J$ such that $(y x)^{n} t \neq 0$. Since $R$ is left AGP-injective, there exists $m>0$ such that $\left((y x)^{n} t\right)^{m} \neq 0$ and $\left((y x)^{n} t\right)^{m} R$ is a direct summand of $\operatorname{rl}\left(\left((y x)^{n} t\right)^{m}\right)$. Write $\left((y x)^{n} t\right)^{m}=(y x)^{n} s$ where $s=t\left((y x)^{n} t\right)^{m-1} \in J$. Then $\mathbf{r l}\left((y x)^{n} s\right)=(y x)^{n} s R \oplus X$ for some right ideal $X$ of $R$. We proceed with the following two cases.

Case 1. $\operatorname{rl}\left((y x)^{n}\right)=\mathbf{r l}\left((y x)^{n} s\right)$. Then $(y x)^{n} \in \mathbf{r l}\left((y x)^{n}\right)=(y x)^{n} s R \oplus X$. Write $(y x)^{n}=(y x)^{n} s v+z$, where $v \in R$ and $z \in X$. Then $(y x)^{n} s=(y x)^{n} s v s+z s$ and 
so $z s \in(y x)^{n} s R \cap X$. Thus, $z s=0$ and hence $(y x)^{n} s=(y x)^{n} s v s$. It follows that $(y x)^{n} s(1-v s)=0$. Since $s \in J, 1-v s$ is a unit in $R$. So, we have $(y x)^{n} s=0$. This is a contradiction.

Case 2. $\mathbf{r l}\left((y x)^{n}\right) \neq \mathbf{r l}\left((y x)^{n} s\right)$. Then $\mathbf{l}\left((y x)^{n}\right) \neq \mathbf{l}\left((y x)^{n} s\right)$. It follows that there exists $u \in \mathbf{I}\left((y x)^{n} s\right)$ but $u \notin \mathbf{I}\left((y x)^{n}\right)$. Thus, $u(y x)^{n} s=0$ and $u(y x)^{n} \neq 0$. This gives that $s \in \mathbf{r}\left(u(y x)^{n}\right)$ and $s \notin \mathbf{r}\left((y x)^{n}\right)$. So, the inclusion $\mathbf{r}\left((y x)^{n}\right) \subset \mathbf{r}\left(u(y x)^{n}\right)$ is proper. This is a contradiction because $0 \neq u(y x)^{n}=\left(u(y x)^{n-1} y\right) x$ and $\mathbf{r}\left(u(y x)^{n}\right) \in \mathscr{F}$.

We have proved that $(y x)^{n} J=0$, and so $R x \cap \mathbf{I}(J) \neq 0$. Therefore, $\mathbf{I}(J)$ is an essential left ideal of $R$.

Next we prove that $J$ is nilpotent. Since $R$ is right noetherian, there exists $k>0$ such that $\mathbf{I}\left(J^{k}\right)=\mathbf{I}\left(J^{k+n}\right)$ for all $n>0$. Suppose $J$ is not nilpotent. Then $J^{k} \neq 0$ and so $M_{R}=R / \mathbf{l}\left(J^{k}\right)$ is a nonzero $R$-module. Since $R$ is right noetherian, the set $\left\{\mathbf{r}_{R}(m): 0 \neq m \in M\right\}$ has a maximal element, $\mathbf{r}_{R}\left(m_{1}\right)$ say. Write $m_{1}=x+\mathbf{I}\left(J^{k}\right)$ where $x \in R$. Then $x J^{k} \neq 0$. Since $\mathbf{l}\left(J^{2 k}\right)=\mathbf{l}\left(J^{k}\right)$, we see $x J^{k} \notin \mathbf{I}\left(J^{k}\right)$. So, there exists $b \in J^{k}$ such that $x b \notin \mathbf{I}\left(J^{k}\right)$. Since $\mathbf{I}(J) \leq_{e} R, R x b \cap \mathbf{l}\left(J^{k}\right) \neq 0$. So, we have $0 \neq a x b \in \mathbf{I}\left(J^{k}\right)$ for some $a \in R$. Let $m_{2}=a x+\mathbf{I}\left(J^{k}\right) \in M$. Then $m_{2} \neq 0$ and $b \in \mathbf{r}_{R}\left(m_{2}\right)$. But, $b \notin \mathbf{r}_{R}\left(m_{1}\right)$. So, the inclusion $\mathbf{r}_{R}\left(m_{1}\right) \subset \mathbf{r}_{R}\left(m_{2}\right)$ is proper. This contradicts the choice of $m_{1}$.

Finally, for any $0 \neq x \in R, x J=0$, or $x J^{n} \neq 0$ and $x J^{n+1}=0$ for some $n>0$. It follows that $x R \cap \mathbf{I}(J) \neq 0$. So, $\mathrm{I}(J)$ is an essential right ideal of $R$.

The next result extends [12, Corollary 2.9]. (Note that, if $R$ is left Kasch, then $R_{R}$ satisfies (C2) (see [25]) and hence satisfies (GC2)).

COROLLARY 2.2. Every right noetherian, left AGP-injective ring $R$ such that $R_{R}$ satisfies $(\mathrm{GC} 2)$ is right artinian.

Proof. Since $R$ is right finitely dimensional and $R_{R}$ satisfies (GC2), $R$ is semilocal by Lemma 1.1. By Theorem $2.1, J(R)$ is nilpotent. So, $R$ is semiprimary. Since $R$ is right noetherian, $R$ is right artinian.

Next, we consider semiprime left AGP-injective rings.

LEMMA 2.3. Let $R$ be an arbitrary ring and $a \in R$ such that $\mathrm{I}(a)$ is a maximal left annihilator or $\mathbf{r}(a)$ is a maximal right annihilator. Then $\mathbf{I}(a t)=\mathbf{I}(a)$ for any $t \notin \mathbf{r}(a)$ and $Z_{l} \subseteq \mathbf{r}(a)$, and $\mathbf{r}(t a)=\mathbf{r}(a)$ for any $t \notin \mathbf{I}(a)$ and $Z_{r} \subseteq \mathbf{l}(a)$.

Proof. Let $x \in Z_{l}$. Then $\mathrm{I}(x)$ is essential in ${ }_{R} R$. So, $\mathrm{l}(x) \cap R r \neq 0$ for any $0 \neq r \in R$. Thus, there exists $y \in R$ such that $0 \neq y r$ and $y r x=0$. So, the inclusion $\mathbf{I}(r) \subset \mathbf{I}(r x)$ is proper. 
Case 1. Let $\mathbf{I}(a)$ be a maximal left annihilator. As above, $\mathbf{I}(a) \subset \mathbf{I}(a x)$ for all $x \in Z_{l}$. It must be that $a x=0$. This shows that $a \in \mathbf{I}\left(Z_{l}\right)$. Clearly, in this case $\mathrm{l}(a t)=\mathrm{l}(a)$ for any $t \notin \mathrm{r}(a)$.

Case 2. Let $\mathbf{r}(a)$ be a maximal right annihilator. If $t \notin \mathbf{r}(a)$, then $a t \neq 0$. For $x \in \mathrm{I}(a t), t \in \mathbf{r}(x a)$ and so the inclusion $\mathbf{r}(a) \subset \mathbf{r}(x a)$ is proper. By the maximality of $\mathbf{r}(a), x a=0$. Thus, $\mathbf{I}(a t)=\mathbf{l}(a)$. It follows that $R a \cap \mathbf{l}(t)=0$. Thus, $t \notin Z_{l}$. Therefore, $Z_{l} \subseteq \mathbf{r}(a)$.

The remaining part is by the left-right symmetry of the hypothesis.

The next theorem extends [7, Theorem 3.1].

THEOREM 2.4. Let $R$ be a semiprime left AGP-injective ring. Then every maximal left (respectively, right) annihilator is a maximal left (respectively, right) ideal of $R$ which is generated by an idempotent.

PRoOF. Let $L$ be a maximal left (respectively, right) annihilator. Then $L=\mathbf{I}(a)$ (respectively, $\mathbf{r}(a)$ ) for some $0 \neq a \in R$. Since $R$ is semiprime, $Z_{l} \cap \mathbf{I}\left(Z_{l}\right)=0$. Claim: $a \notin Z_{l}$. Otherwise, $a \notin \mathbf{l}\left(Z_{l}\right)$, that is, $a Z_{l} \neq 0$. Take $x \in Z_{l}$ such that $a x \neq 0$. Since $x \notin \mathbf{r}(a), \mathrm{I}(a x)=\mathbf{I}(a)$ by Lemma 2.3. Thus, $\mathrm{I}(x) \cap R a=0$, a contradiction, since $x \in Z_{l}$. Therefore, $a \notin Z_{l}$. By Lemma 1.3 and Lemma 1.4, the inclusion $\mathrm{I}(a) \subset \mathrm{I}(a-a r a)=\mathrm{I}[a(1-r a)]$ is proper for some $r \in R$. It follows from Lemma 2.3 that $a-a r a=0$. Therefore, $L=\mathrm{l}(a r)$ (respectively, $L=\mathbf{r}(r a)$ ) with ar (respectively, $r a$ ) an idempotent. So we can assume that $a=e$ is an idempotent. To see $L$ is a maximal left (respectively, right) ideal, we show that $R e$ (respectively, $e R$ ) is a minimal left (respectively, right) ideal of $R$. Since $R$ is semiprime, it suffices to show that $e R e$ is a division ring. Let $0 \neq d \in e R e$. Since $R$ is left AGP-injective, there exists $n>0$ such that $d^{n} \neq 0$ and $d^{n} R$ is a direct summand of $\operatorname{rl}\left(d^{n}\right)$. By Lemma 2.3, $\mathbf{l}\left(d^{n}\right)=\mathbf{l}(e)$ and so $\mathbf{r l}\left(d^{n}\right)=\mathbf{r l}(e)=e R$. Thus, $d^{n} R$ is a direct summand of $e R$ and hence of $R_{R}$. It follows that $d^{n} R=\operatorname{rl}\left(d^{n}\right)=e R$. Write $e=d^{n} b$ where $b \in R$. Then $e=d\left(d^{n-1} b e\right)$ with $d^{n-1} b e \in e R e$. So, $e R e$ is a division ring.

A ring $R$ is a left PP ring if every principal left ideal of $R$ is projective. The next result extends [6, Theorem 2.9] from a left GP-injective ring to a left AGP-injective ring.

PROPOSITION 2.5. The ring $R$ is a von Neumann regular ring if and only if $R$ is left $P P$ and left AGP-injective.

PROOF. One direction is obvious. Suppose that $R$ is left PP and left AGP-injective. For any nonzero element $a \in R$, there exists $n>0$ such that $a^{n} \neq 0$ and $\mathbf{r l}\left(a^{n}\right)=$ $a^{n} R \oplus X$ where $X$ is a right ideal of $R$. Since $R$ is left PP, $R a^{n}$ is projective, and 
hence $0 \rightarrow \mathrm{I}\left(a^{n}\right) \rightarrow R \rightarrow R a^{n} \rightarrow 0$ splits. Thus, $\mathbf{I}\left(a^{n}\right)=R e$ where $e^{2}=e \in R$. It follows that $\mathbf{r l}\left(a^{n}\right)=\mathbf{r}(R e)=(1-e) R$. Thus, $a^{n} R$ is a direct summand of $(1-e) R$, and hence a direct summand of $R_{R}$. This implies that $a^{n}$ is a regular element of $R$. If $a \neq 0$ but $a^{2}=0$, the argument above shows that $a$ is a regular element. So, by [6, Theorem 2.9], $R$ is a regular ring.

\section{Right quasi-dual rings}

Following [21], a ring $R$ is called right quasi-dual if every right ideal of $R$ is a direct summand of a right annihilator. As shown in [21], the ring $R$ is right quasi-dual if and only if every essential right ideal of $R$ is a right annihilator if and only if every singular cyclic right $R$-module is cogenerated by $R$. Every right dual ring is certainly right quasi-dual, and every right quasi-dual ring is left AP-injective.

LEMMA 3.1. Let $R$ be a right quasi-dual ring. For any right ideal $I$ of $R$ and $a \in R, \mathbf{r}[R a \cap \mathbf{I}(I)]=I+\left(X_{a l}: a\right)_{r}$ with $\left(X_{a l}: a\right)_{r} \cap I \subseteq \mathbf{r}(a)$ and $\left(X_{a l}: a\right)_{r}=$ $\left\{x \in R: a x \in X_{a l}\right\}$, where $X_{a l}$ is a right ideal of $R$ such that $\mathbf{r l}(a I)=a I \oplus X_{a l}$.

PROOF. Let $x \in \mathbf{r}[R a \cap \mathbf{l}(I)]$. Then $\mathbf{l}(a I) \subseteq \mathbf{l}(a x)$, and so $a x \in \mathbf{r l}(a x) \subseteq \mathbf{r l}(a I)=$ $a I \oplus X_{a l}$. Write $a x=a t+y$ where $t \in l$ and $y \in X_{a l}$. Then $a(x-t)=y \in X_{a l}$ and thus $x-t \in\left(X_{a l}: a\right)_{r}$. Therefore, $x \in I+\left(X_{a l}: a\right)_{r}$ and $\mathbf{r}[R a \cap l(I)] \subseteq I+\left(X_{a l}: a\right)_{r}$. It is easy to see that $\left(X_{a l}: a\right)_{r} \cap I \subseteq \mathbf{r}(a)$ and that $I \subseteq \mathbf{r}[R a \cap \mathbf{l}(I)]$. Let $y \in\left(X_{a l}: a\right)_{r}$. Then $a y \in X_{a l} \subseteq \mathbf{r l}(a l)$. For any $r a \in R a \cap \mathbf{l}(I)$, $r a I=0$. This gives that $r \in \mathbf{l}(a I)$. Since $a y \in \mathbf{r l}(a I)$, it follows that $r a y=0$. Thus, $y \in \mathbf{r}[R a \cap \mathbf{I}(I)]$ and $\left(X_{a I}: a\right)_{r} \subseteq \mathbf{r}[R a \cap \mathbf{l}(I)]$.

THEOREM 3.2. Let $R$ be a right quasi-dual ring and $J=J(R)$. Then

(1) $J=Z_{l}=\mathbf{r}\left(S_{r}\right), S_{r}=\mathbf{r}\left(Z_{r}\right)$, and $R$ is right Kasch.

(2) $\mathbf{I}(J)$ is essential in ${ }_{R} R$.

PROOF. (1). Clearly, $S_{r} \subseteq \mathbf{r}\left(Z_{r}\right)$. Let $K$ be any essential right ideal of $R$. Then $\mathbf{l}(K) \subseteq Z_{r}$ and so $K=\mathbf{r l}(K) \supseteq \mathbf{r}\left(Z_{r}\right)$. It follows that $S_{r} \supseteq \mathbf{r}\left(Z_{r}\right)$ since $S_{r}$ is the intersection of all essential right ideals. Thus, $S_{r}=\mathbf{r}\left(Z_{r}\right)$. By [21, Lemma 2.5 and Lemma 2.6], $J=Z_{l}$ and $R$ is right Kasch. Since $R$ is right Kasch, $J=\mathbf{r}\left(S_{r}\right)$.

(2). Let $0 \neq a \in R$ and assume that $R a \cap \mathbf{I}(J)=0$. Then, by Lemma 3.1, $R=\mathbf{r}[R a \cap \mathbf{I}(J)]=J+\left(X_{a J}: a\right)_{r}$ where $X_{a J}$ is a right ideal of $R$ such that $\operatorname{rl}(a J)=a J \oplus X_{a J}$. Since $J$ is small in $R_{R}, R=\left(X_{a J}: a\right)_{r}$. It follows that $a R \subseteq X_{a J}$ and so $a J \subseteq a J \cap X_{a J}=0$. Thus, $a \in R a \cap \mathbf{I}(J)=0$, a contradiction.

COROLlaRY 3.3. Let $R$ be a quasi-dual ring. Then $S=S_{r}=S_{l}$ is essential as a left and a right ideal of $R$. 
ProOF. By [21, Theorem 2.8] and Theorem 3.2.

It was proved in [21] that, for a two-sided quasi-dual ring $R$, every Goldie torsion right $R$-module is cogenerated by $R_{R}$ if and only if the second singular right ideal $Z_{2}\left(R_{R}\right)$ of $R$ is injective. This result can be improved as follows.

THEOREM 3.4. Consider the following conditions on a ring $R$ :

(1) Every Goldie torsion right $R$-module is cogenerated by $R_{R}$.

(2) $Z_{2}\left(R_{R}\right)$ is injective and $R$ is right Kasch.

(3) $R$ is right self-injective and right Kasch.

Then (3) implies (2) and (2) implies (1). In additton (1) implies (3) if $R$ is left quasi-dual.

ProOF. (3) implies (2) is obvious, and (2) implies (1) is by the proof of [21, Theorem 4.1].

Suppose $R$ is left quasi-dual and (1) holds. By [21, Theorem 4.1], $Z_{2}\left(R_{R}\right)$ is injective. Write $R_{R}=Z_{2}\left(R_{R}\right) \oplus K$ where $K$ is right ideal of $R$. It suffices to show that $K_{R}$ is injective. Note that $R$ is a two-sided quasi-dual ring, so $Z_{l}=Z_{r}$ and $S_{r}=\mathbf{l}\left(Z_{l}\right)$ by [21, Theorem 2.8]. It follows that $K \subseteq \mathrm{l}\left(\left(Z_{2}\left(R_{R}\right)\right) \subseteq \mathrm{l}\left(Z_{l}\right)=S_{r}\right.$. So, $K_{R}$ is semisimple. Thus, to show that $K_{R}$ is injective, it suffices to show that $K$ is $Z_{2}\left(R_{R}\right)$-injective. But, this is clear because $K$ is non-singular and $Z_{2}\left(R_{R}\right)$ is Goldie torsion.

A ring $R$ is right PF if $R$ is an injective cogenerator for Mod- $R$. It is known that $R$ is right PF if and only if $R$ is right self-injective and right Kasch. The next corollary improved [21, Corollaries 4.4-4.6].

COROLLARY 3.5. $R$ is a two-sided $P F$-ring if and only if every Goldie torsion right $R$-module is cogenerated by $R_{R}$ and every Goldie torsion left $R$-module is cogenerated by ${ }_{R} R$.

Dischinger and Müller [8] constructed a left PF-ring that is not right PF. By Corollary 3.5, the left PF-ring in [8] does not cogenerate every Goldie torsion right $R$ module. Osofsky [19] constructed a non-injective cogenerator for Mod- $R$. We note that Osofsky's ring $R$ has the property that $Z_{2}\left(R_{R}\right)=R$ (since $J(R)^{2}=0$ and $\left.J(R)_{R} \leq_{e} R_{R}\right)$. This shows the conditions (1) and (2) in Theorem 3.4 are not equivalent.

PROPOSITION 3.6. The following are equivalent for a ring $R$ :

(1) $R$ is right $P F$.

(2) $Z_{2}\left(R_{R}\right)$ is injective, $R$ is right Kasch and $R=Z_{2}\left(R_{R}\right)+S_{r}$. 
PROOF. (2) implies (1). It suffices to show that $R$ is right self-injective. Since $R=Z_{2}\left(R_{R}\right)+S_{r}, R=Z_{2}\left(R_{R}\right) \oplus K$ where $K$ is a non-singular semisimple right ideal of $R$. Clearly, $K_{R}$ is $Z_{2}\left(R_{R}\right)$-injective and $K_{R}$-injective. So, $K_{R}$ is injective. Thus, $R_{R}$ is injective.

(1) implies (2). We only need to show that $R=Z_{2}\left(R_{R}\right)+S_{r}$. Since $Z_{2}\left(R_{R}\right)$ is injective, write $R=Z_{2}\left(R_{R}\right) \oplus K$ where $K$ is a right ideal of $R$. Since $R$ is right PF, $J(R)=Z_{r} \subseteq Z_{2}\left(R_{R}\right)$ and $S_{r}$ is a finitely generated essential right ideal of $R$. Thus $\operatorname{Soc}\left(K_{R}\right)$ is finitely generated and essential in $K_{R}$. Since every minimal right ideal contained in $K$ is idempotent, $\operatorname{Soc}\left(K_{R}\right)$ is a summand of $R_{R}$ and hence of $K_{R}$. Thus, $K=\operatorname{Soc}\left(K_{R}\right)$ is semisimple.

We do not know if the condition that $R=Z_{2}\left(R_{R}\right)+S_{r}$ in Proposition 3.6 can be removed.

\section{Acknowledgment}

The author is very grateful to the referee for careful reading this article and valuable suggestions, in particular, the comments on weakening the hypothesis in Corollary 1.8.

\section{References}

[1] G. Azumaya, 'Finite splitness and finite projectivity', J. Algebra 106 (1987), 114-134.

[2] J. E. Björk, 'Rings satisfying certain chain conditions', J. Reine Angew. Math. 245 (1970), 63-73.

[3] V.Camillo, 'Commutative rings whose principal ideals are annihilators', Portugal. Math. 46 (1989), 33-37.

[4] V. Camillo and M. F. Yousif, 'Continuous rings with ACC on annihilators', Canad. Math. Bull. 34 (1991), 462-464.

[5] R. Camps and W. Dicks, 'On semi-local rings', Israel J. Math. 81 (1993), 203-211.

[6] J. Chen and N. Ding, 'On regularity of rings', Algebra Colloq. 8 (2001) 267-274.

[7] — 'On general principally injective rings', Comm. Algebra 27 (1999), 2097-2116.

[8] F. Dischinger and W. Müller, 'Left PF is not right PF', Comm. Algebra 14 (1986), 1223-1227.

[9] C. Faith, 'Rings with ascending chain conditions on annihilators', Nagoya Math. J. 27 (1966), 179-191.

[10] C. Faith and P. Menal, 'A counter example to a conjecture of Johns', Proc. Amer. Math. Soc. 116 (1992), 21-26.

[11] _ - 'The structure of Johns rings', Proc. Amer. Math. Soc. 120 (1994), 1071-1081.

[12] J. L. Gómez Pardo and P. A. Guil Asensio, 'Torsionless modules and rings with finite essential socle', in: Abelian groups, module theory, and topology (Padua, 1997) (eds. D. Dikranjan and L. Salce), Lecture Notes in Pure and Appl. Math. 201 (Dekker, New York, 1998) pp. 261-278.

[13] K. R. Goodearl, Von Neumann regular rings (Pitman, London, 1979).

[14] B. Johns, 'Annihilator conditions in noetherian rings', J. Algebra 30 (1974), 103-121.

[15] S. B. Nam, N. K. Kim and J. Y. Kim, 'On simple GP-injective modules', Comm. Algebra 23 (1995), 5437-5444. 
[16] W. K. Nicholson and M. F. Yousif, 'On a theorem of Camillo', Comm. Algebra 23 (1995), 53095314.

[17] —_- 'Principally injective rings', J. Algebra 174 (1995), 77-93.

[18] - 'Mininjective rings', J. Algebra 187 (1997), 548-578.

[19] B. L. Osofsky, 'A generalization of quasi-Frobenius rings', J. Algebra 4 (1966), 373-387.

[20] S. Page and Y. Zhou, 'Generalizations of principally injective rings', J. Algebra 206 (1998), 706-721.

[21] - 'Quasi-dual rings', Comm. Algebra 28 (2000), 489-504.

[22] G. Puninski, R. Wisbauer and M. F. Yousif, 'On P-injective rings', Glasgow Math. J. 37 (1995), 373-378.

[23] E. A. Rutter, 'Rings with the principal extension property', Comm. Algebra 3 (1975), 203-212.

[24] W. Xue, 'A note on YJ-injectivity', Riv. Mat. Univ. Parma (6) 1 (1998), 31-37 (1999).

[25] M. F. Yousif, 'CS rings and Nakayama permutations', Comm. Algebra 25 (1997), 3787-3795.

[26] R. Yue Chi Ming, 'On injectivity and p-injectivity', J. Math. Kyoto Univ. 27 (1987), 439-452.

Department of Mathematics and Statistics

Memorial University of Newfoundland

St. John's A1C 5S7

Canada

e-mail: zhou@math.mun.ca 